\title{
RDUS
}

Revue de DROIT UNIVERSITÉ DE SHERBROOKE

Titre : $\quad$ LES MÉDIAS ÉCRITS ET LE PROCESSUS D'ÉMERGENCE DE LA LOI 120

Auteur(s): Daniel DUCHARME, Anne MARCOUX, Guy ROCHER, Andrée LAJOIE

Revue : $\quad$ RDUS, 1997-1998, volume 28, numéro 1-2

Pages: $\quad 125-150$

ISSN : $\quad 0317-9656$

Éditeur : $\quad$ Université de Sherbrooke. Faculté de droit.

URI : $\quad$ http://hdl.handle.net/11143/12739

DOI : $\quad$ https://doi.org/10.17118/11143/12739 
Page vide laissée intentionnellement. 


\section{LES MÉDIAS ÉCRITS ET LE PROCESSUS D'ÉMERGENCE DE LA LOI 120}

par Daniel DUCHARME* Anne MARCOUX** Guy ROCHER *** Andrée LAJOIE****

SOMMAIRE

INTRODUCTION

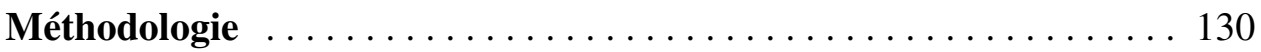

Le constat d'une surcharge bureaucratique $\ldots \ldots \ldots \ldots \ldots 131$

Un partenariat médecins-journalistes contre l'ennemi commun : la

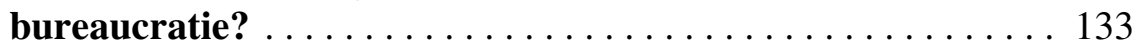

Un point tournant : la campagne publicitaire des médecins . . . . . 134

L'insertion de points de vue alternatifs dans le discours médiatique : le cas de la Coalition pour la santé et le bien-être . . . . . . . . . 139

*. Sociologue, Centre de recherche en droit public, Faculté de droit, Université de Montréal.

**. $\quad$ Avocate-conseil, Conseil de la Santé et du Bien-Être du Québec.

***. Professeur titulaire et chercheur, Centre de recherche en droit public, Faculté de droit, Université de Montréal.

****. Professeure titulaire et chercheure, Centre de recherche en droit public, Faculté de droit, Université de Montréal. 
Les négociations de l'été 1991 et le retour de l'usager dans les quotidiens québécois $\ldots \ldots \ldots \ldots \ldots \ldots \ldots \ldots \ldots \ldots \ldots \ldots \ldots \ldots \ldots \ldots \ldots \ldots \ldots \ldots \ldots$

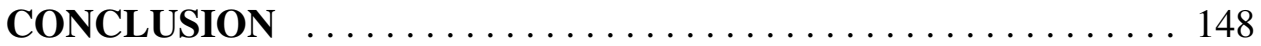




\section{INTRODUCTION}

Le processus d'élaboration d'une loi aussi importante que celle qui concerne les Services de Santé et les Services sociaux monopolise l'attention d'un nombre impressionnant de groupes sociaux qui s'affrontent sur la base d'intérêts divergents. De façon à inscrire ces intérêts dans un éventuel corpus juridique formalisé, les acteurs sociaux qui participent à l'élaboration d'une telle loi multiplient les stratégies pour mettre de l'avant l'univers normatif qu'ils privilégient.

L'affrontement des ordres normatifs qui en résulte se joue donc à plusieurs niveaux. Pour s'enfoncer plus loin dans la métaphore théâtrale, nous pourrions même dire qu'il s'articule autour de scènes sur lesquelles interagissent les acteurs sociaux. C'est de la capacité à s'emparer de ces scènes que dépend le succès de l'entreprise de mobilisation et de défense des intérêts des groupes sociaux impliqués dans ce débat législatif. Rien n'est donc laissé au hasard pour les groupes qui veulent faire inscrire leurs droits dans un projet de loi - il faut à tout prix que leurs voix soient entendues sur toutes ces scènes afin que leurs intérêts trouvent écho dans le texte législatif à venir.

Les scènes d'intervention des acteurs sociaux impliqués dans ce processus législatif ne sont pas légion. Nous pouvons déjà en distinguer quelques unes qui sont incontournables : la scène privée où interagissent les groupes professionnels et les groupes d'intérêts, la scène publique où ce sont les membres de la classe politique et de la fonction publique qui interagissent entre eux et finalement, la scène politique où les membres des deux scènes mentionnées précédemment se rejoignent pour créer un véritable débat législatif. Il existe toutefois une scène qui semble cristalliser toutes les autres en réunissant dans un débat encore plus large les univers du privé et du public, c'est la scène médiatique, qui fait entrer le reste de la société civile dans le processus législatif à travers la mission d'information de l'opinion publique que les médias instaurent.

Il n'est pas trop de dire que cette dernière scène occupe un rôle essentiel dans le débat qui entoure l'élaboration d'une nouvelle législation. Qui plus est, elle occupe une place primordiale dans l'esprit des groupes qui veulent informer et influencer l'opinion publique de façon à ce que celle-ci intercède en leur 
faveur auprès du législateur. Les stratégies sont nombreuses pour que les journalistes fassent écho aux intérêts des groupes impliqués dans la discussion entourant un projet de loi : conférences et points de presse, entrevues, abondante documentation livrée aux médias, campagne de publicité, etc...

Si la réaction de la population profane à un projet de loi est en général fortement influencée par ces contenus médiatiques, il est à se demander toutefois si cette réaction est bel et bien le résultat d'un processus d'information du public ou si elle ne serait pas plutôt le fruit d'une manipulation de celui-ci à des fins strictement utilitaristes. La question peut sembler anodine, mais elle n'en demeure pas moins fondamentale. Dans la mesure où les médias produisent eux aussi une normativité qui taille sa place dans le sens commun, il n'est pas faux d'affirmer que le discours médiatique contribue à construire ou à modifier le droit formel. Loin de représenter le discours du citoyen ordinaire, le contenu médiatique se construit souvent à l'insu de ce dernier en prenant tout bonnement le relais du discours officiel de l'État ou des groupes de pression. En ce sens, les journalistes ne laissent s'exprimer que les acteurs déjà présents sur l'arène politique, délaissant par ailleurs ceux dont la voix a le plus besoin de se faire entendre : les citoyens ordinaires qui sont eux aussi interpellés par le nouveau projet de loi.

La couverture médiatique des débats entourant la discussion et l'adoption de la Loi sur les Services de Santé et les Services sociaux est instructive à cet égard. Jamais n'aura-t-on assisté à une mise à l'écart aussi évidente du citoyen que le discours bureaucratique plaçait pourtant, dans une savante rhétorique, au centre de la réforme. ${ }^{1}$ Le survol rapide des grands titres des quotidiens dans la

1. Sur ce point, et pour dissiper toute forme d'équivoque que pourrait entraîner une lecture partisane de cette réforme, nous mentionnerons que nous n'avons pas voulu prendre position en faveur d'aucune catégorie d'acteurs sociaux présents dans le débat. Si certains peuvent voir dans la retranscription des propos de hauts-fonctionnaires, de journalistes, de juristes ou de représentants de groupes professionnels une adhésion naïve et aveugle à leurs propos, nous préférons dire que nous nous en tenons aux principes de la rigueur méthodologique en analyse de discours en sociologie. Sachant que notre corpus de recherche (le contenu des médias écrits) est essentiellement composé de discours (et qu'aucun acteur social n'échappe à la production de discours), nous cherchons plutôt à déterminer quelles en sont les propriétés empiriques constituantes qui permettent de révéler la nature sociale du processus d'émergence de la loi 120. Ainsi, nous pensons établir les bases d'une véritable sociologie 
période qui précède l'adoption de cette loi pourrait toutefois laisser croire le contraire. S'il existe une catégorie d'acteurs du système de santé dont le nom revient tel une rengaine dans les médias écrits, c'est bien celle du citoyen. Pourtant, ce dernier est très rarement considéré comme un élément actif du système de santé ${ }^{2}$ par les journalistes qui font la couverture de ce débat. C'est pourquoi il est très rarement interrogé.

Alors, comment expliquer l'omniprésence du citoyen dans le discours journalistique quand celui-ci n'a pratiquement pas droit au chapitre quand vient le temps de laisser la parole aux acteurs du système de santé? Une piste qui pourrait nous être utile dans la compréhension de ce phénomène inusité est la présence récurrente de la relation entre le patient et le médecin dans les écrits journalistiques que nous avons recensés. Il existe, en effet, un consensus assez fort chez les membres de la presse écrite pour dire que c'est cette relation qui doit servir de balise pour le projet de réforme législative qu'initie le gouvernement québécois. Mais, lorsqu'il s'agit de traiter de ce rapport dialectique, seul le discours du médecin est considéré. Ce qui fait en sorte que la relation patient-médecin, bien qu'elle soit formellement énoncée dans les médias écrits, n'en demeure pas moins présente que de façon virtuelle. Elle est ni plus ni moins remplacée par une dialectique médecins/État qui elle, est bien réelle, et renvoie le citoyen au rang d'une variable muette avec laquelle chaque catégorie d'acteurs jongle, les journalistes étant bien sûr inclus dans cette dynamique.

compréhensive, au sens wébérien du terme, qui nous permettra de mettre à jour les interactions sociales qui participent à l'élaboration du droit formel. Par cela, nous évitons, pensons-nous, le piège (tentant, il va s'en dire) de la pure spéculation sur les véritables motivations cachées derrière le discours des acteurs sociaux mis à l'étude.

2. Et ce malgré le fait que le Ministère de la Santé et des Services sociaux semble vouloir bâtir son discours en lui réservant un tout autre rôle, en le considérant en tant que citoyen payeur, consommateur et décideur. 


\section{Méthodologie}

La revue de presse que nous avons effectuée couvre la période qui s'étend du 1er janvier 1991 au 28 août 1991, date de l'adoption de la loi 120. ${ }^{3}$ Nous avons choisi cette période car elle constitue l'étape ultime du processus d'émergence normative que nous voulons étudier. Le premier semestre de l'année 1991 a vu, en effet, se matérialiser les discussions suite au dépôt du projet de loi à la fin de l'année précédente. Une période intensive de négociations entre les groupes d'intérêts et le gouvernement s'est alors mise en branle et a trouvé son point culminant dans les négociations privées entre la Corporation professionnelle des médecins, les divers syndicats médicaux et le Ministère de la Santé et des Services sociaux à l'été 1991.

Il est inutile de dire que cette période fut particulièrement fertile pour les médias qui couvraient jusqu'alors les principaux développement de cette importante réforme du réseau public de la santé et des services sociaux au Québec. Nous avons donc trouvé là un terrain fécond pour l'étude du rôle des médias dans le processus d'émergence de la loi 120.

Pour les fins de notre enquête, nous avons retenu environ 1000 articles qui proviennent principalement de trois grands quotidiens montréalais : La Presse, Le Devoir et The Gazette. Nous avons aussi tenu compte des quotidiens régionaux (Le Soleil, Journal de Québec, La Tribune, ...), qui reflètent parfois des préoccupations différentes de celles qui sont soulevées dans la métropole. Dans cette vaste revue de presse, notre attention a surtout porté sur les articles d'opinion, les billets et les éditoriaux qui traduisent mieux les grandes orientations et prises de position que souhaitent prendre les divers quotidiens dans l'élaboration de ce projet législatif. Cela ne veut pas dire pour autant que nous avons négligé les articles informatifs plus classiques dans notre travail de dépouillement des quotidiens québécois. Ces derniers nous sont apparus pertinents dans la mesure où ils nous informaient sur les principaux acteurs qui ont accès à la scène médiatique, ceux qui y ont droit de parole et peuvent, par

3. Nous avons ajouté à notre corpus une série d'une quinzaine d'articles, publiés entre le 28 août 1991 et le 15 septembre 1991, qui font le post-mortem des débats sur le projet de loi 120. 
le fait même, exercer une certaine influence sur l'opinion publique. Ces observations nous permettent aussi de déterminer quels sont les exclus de cette même scène, ceux dont la parole n'est pas prise en compte, pour un motif ou pour un autre.

Un premier tour d'horizon de cette abondante littérature nous a permis de constater l'omniprésence des groupes médicaux dans la presse écrite. Plus de $80 \%$ des articles (825 sur 1013 , soit environ $81,44 \%$ du corpus) mettent en scène les médecins, alors que seulement $40 \%$ traitent de la situation des usagers (397 sur 1 013, 39,19\% du corpus). On parle donc deux fois moins dans les journaux montréalais des bénéficiaires des soins de santé que de ceux qui les prodiguent.

\section{Le constat d'une surcharge bureaucratique}

Et pourtant, certains éditorialistes et columnistes (peu nombreux, il faut l'admettre) s'accordent avec les objectifs proposés dans le discours du Ministère de la Santé et des Services sociaux pour placer le citoyen au centre du système de soins. C'est du moins ce qui ressort de la lecture de certains articles rédigés dans les mois qui suivirent le dépôt du projet de loi à la fin de l'année 1990. Quelques journalistes défendront avec beaucoup de vigueur le droit des usagers en venant à la rescousse du principe de l'accessibilité universelle aux soins de santé, considérant que les citoyens seront affectés dans ce projet de loi «[...] par l'érosion graduelle des deux principes fondamentaux du régime d'assurancesanté : la gratuité et l'accessibilité.» ${ }^{4}$ Certains journalistes anglophones s'insurgeront même contre le Ministère de la Santé et des Services sociaux en mentionnant que l'idée du citoyen au centre du système n'est que pure démagogie, car dans les faits, celui-ci se trouve pratiquement écarté de ce système dans l'esprit du projet de loi 120 qui propose une réforme de structures bureaucratiques plutôt que d'idées :

«The real danger is that bureaucratic structures will impede rather than enhance access to medicare for ordinary Quebecers and perhaps particularly for anglophone Quebecers, even though their right for

4. L Gagnon, «L'érosion du régime», La Presse [de Montréal] (8 juin 1991) B3. 
health and social services in their own language is reaffirmed in Bill $120(\ldots)$ the patient (...) is not served well by such a fragmented, overstructured system.» ${ }^{5}$

Le constat d'une surcharge bureaucratique du système de santé est partagé par le corps médical qui prend ici toute la place qui lui est offerte dans les médias pour critiquer les visées gestionnaires de l'État québécois. Le Dr. Jacques Genest, fondateur de l'Institut de recherche clinique de Montréal, écrira dans La Presse du 31 janvier 1991, que le Livre Blanc du ministre Côté est le produit de la réflexion de hauts-fonctionnaires qui nourrissent de l'antagonisme à l'égard des médecins et qui désirent contrôler l'ensemble du système de santé québécois :

«Dans son livre blanc intitulé Une réforme axée sur le citoyen, le ministre du MSSS, Monsieur Marc-Yvan Côté prend, malgré d'excellents objectifs et des recommandations souvent justes, des risques sérieux. On mentionne que le Livre blanc a été préparé par une quarantaine de fonctionnaires du MSSS qui, malgré leurs bonnes intentions, n'ont pas suffisamment de perception de la réalité médicale et chez qui ont sent, à tort ou à raison, un certain antagonisme à l'endroit de la profession médicale et une volonté de contrôle. La lecture du document fait voir une technocratie envahissante qui laisse de côté les aspects humains de la médecine.» ${ }^{6}$

Cet avis est aussi partagé par Lysiane Gagnon du quotidien La Presse qui dénonce l'omniprésence de la fonction publique dans le projet de réforme de la santé et des services sociaux. Elle fait remarquer qu'à l'exception de Jean Rochon, tous les principaux acteurs qui ont participé à l'élaboration de cette réforme depuis la Commission d'Enquête sur les Services de Santé et les Services sociaux jusqu'à l'élaboration de l'avant-projet de loi 120 occupaient des postes au sein du réseau des Affaires sociales. «C'est dire que le système se jugeait lui-même. Rien d'étonnant à ce que la Commission Rochon ait recommandé l'accroissement des pouvoirs... des administrateurs!» ${ }^{7}$

5. «Shelve Health Reform Bill», The Gazette [de Montréal] (7 juin 1991) B2.

6. J. Genest, «Attention, M. le Ministre!», La Presse [de Montréal] (31 janvier 1991) B3.

7. L. Gagnon, «L'exclusion des médecins», La Presse [de Montréal] (28 mai 1991) B3. 
(1997-98) 28 R.D.U.S.

Les médias écrits

et le processus d'émergence

\section{Un partenariat médecins-journalistes contre l'ennemi commun : la bureaucratie?}

Jean Paré, du mensuel L'Actualité, ajoute sa voix à celle de Lysiane Gagnon et de nombreux autres journalistes pour dénoncer la quête de légitimité et de pouvoir des bureaucrates :

«C'est une lutte de pouvoir, de contrôle social. Les bureaucrates sont des gens frustrés : leur pouvoir n'est que délégué, et ils en veulent à tout ce qui est autonome : politiciens, journalistes, professionnels, médecins, gens d'affaires... Il y a lutte constante, et constant mouvement de balancier. Aujourd'hui, l'Est tente de sortir de l'illusion étatique. D'autres s'y précipitent. [...] La guerre est déclarée entre les classes d'une nomenklatura qui se dispute le pouvoir et ses retombées.» ${ }^{8}$

La lecture des différents quotidiens de la province démontre bien que les médias écrits rejoignent souvent le discours des médecins pour dénoncer les excès de la bureaucratie. Ainsi, le docteur Robert Marier, président de la Fédération des médecins spécialistes du Québec (FMSQ), rapporte, par exemple, à une journaliste du Journal de Québec :

«C'est bizarre, le ministre est correct lorsque nous le rencontrons, mais on dirait qu'il n'est pas capable de faire traduire ses propos pour améliorer la réforme par ses technocrates $(. ..){ }^{9}$

De nombreux écrits de sociologues, d'économistes ou de spécialistes en matière de droit de la santé tapisseront les pages des grands quotidiens québécois et viendront même renforcer cette charge anti-bureaucratie.

Il existe certes des exemples de points de vue divergents mais ils sont peu nombreux et ne bénéficient souvent pas d'une bonne visibilité dans la mise en page des grands quotidiens québécois. L'un des rares détracteurs de la

8. J. Paré, «Les médecins ou les technocrates?», La Tribune [de Sherbrooke] (3 janvier 1991) B-2.

9. M. Coudé-Lord, «Le lobbying des médecins multiplie les pressions», Journal de Québec (28 mai 1991) 12. 
querelle entre les médias et la bureaucratie (ou encore des médecins et de la bureaucratie) est sans contredit l'éditorialiste Jean Francoeur qui considère que le développement important des technologies médicales a transformé bon nombre d'hôpitaux en entreprises complexes et de grandes tailles, ce qui a rendu nécessaire une bureaucratie administrative. Il reconnaît donc que le système s'est bureaucratisé tant au niveau des établissements que du ministère mais n'y voit là aucune cause de panique, seulement une réalité normale de toute société avancée, «qu'elles soient de type capitaliste ou soviétique!». ${ }^{10}$

\section{Un point tournant : la campagne publicitaire des médecins}

L'apparente unanimité qui semble lier les médecins aux médias dans leur critique du pouvoir bureaucratique s'éteindra toutefois avec l'avènement de la campagne publicitaire du corps médical contre la réforme du ministre Côté au printemps de 1991. Parmi les messages publicitaires que l'on retrouve à cette époque dans les grands quotidiens québécois, un a particulièrement attiré l'attention. Il s'agit d'une photo d'une jeune personne inanimée et couchée sur une civière au dessus de laquelle on peut lire : «Après le 21 juin, sa vie reposera entre les mains des bureaucrates.» Puis dans le texte situé au bas de cette photo, nous lisons :

«Le Québec est reconnu pour avoir l'un des meilleurs systèmes de santé du monde... pourtant, aujourd'hui, la situation est critique.

En effet, notre système de santé est sérieusement menacé par le projet de loi 120 du ministre Côté. C'est une réforme inacceptable, centrée sur l'appareil bureaucratique. Une réforme qui mettra en péril, entre autres, la qualité des soins, la confidentialité de votre dossier et le droit de choisir votre médecin et votre centre hospitalier.

Pire encore, le ministre Côté persiste à vouloir faire adopter, d'ici le 21 juin, ce projet de loi bâclé et rédigé à la hâte. 
Appuyons nos médecins et rejetons la loi 120. Notre santé est en jeu et il est préférable qu'elle soit entre les mains d'un médecin plutôt qu'entre celles d'un bureaucrate.»

La Corporation professionnelle des médecins publie, quant à elle, une publicité en son propre nom où elle interpelle directement la population québécoise :

«Citoyens et citoyennes du Québec

Votre dossier médical ou social vous appartient!

Jusqu'à quand?

... Si vous ne voulez pas risquer que des informations sur vos problèmes passés ou actuels, vos maladies, vos opérations, vos traitements puissent être utilisées sans votre consentement, réagissez tout de suite. Dites non au projet de loi 120.»

Cette campagne publicitaire des médecins suscitera chez les journalistes de nombreuses réactions négatives. Isabelle Paré du Devoir écrira, par exemple :

«Publicité-choc ou démagogie? Placardée à pleines pages dans les quotidiens québécois, la pub choisie par les médecins pour dénoncer le projet de réforme de la santé a semé, hier, la controverse jusqu'aux portes de l'Assemblée nationale.» ${ }^{11}$

Certains diront même que la démagogie médicale se substitue à ce moment à la démagogie de l'État. Pour la première fois dans les quotidiens québécois, on assiste à une ouverture des journalistes au discours des hautsfonctionnaires et des politiciens. C'est ainsi que les médias accorderont une grande importance aux propos de Marc-Yvan Côté émis lors d'un point de presse réalisé à Québec, le 7 juin 1991. Le ministre déclarera à ce moment que la publicité des médecins est indécente et mensongère et enjoindra la population québécoise à ne pas croire à cette offensive «malsaine» du corps médical. ${ }^{12}$

11. I. Paré, «Un placard qui choque», Le Devoir [de Montréal] (8 juin 1991) A1.

12. Presse Canadienne, «Côté pourrait favoriser un large débat public», Journal de Québec (8 juin 1991) 10; D. Lessard, «Côté s'en prend aux tactiques indécentes des médecins», $L a$ Presse [de Montréal] (8 juin 1991) A1; Presse Canadienne, «Marc-Yvan Côté dénonce le dernier message publicitaire», Le Soleil [de Québec] (8 juin 1991) A3. 
Mentionnons que la campagne publicitaire des médecins s'est aussi étendue à la télévision où Radio-Québec et Radio-Canada ont refusé de diffuser leur message qui a été qualifié de message qui porte à controverse. L'affaire a été largement débattue dans les médias écrits qui ont, pour l'ensemble, cautionné la décision des deux réseaux publics de télévision. ${ }^{13}$ Josée Boileau fait, dans un de ses billets, une description des plus complètes de cette publicité :

«Coeurs sensibles, prière de s'accrocher : la guerre des médecins contre la réforme de la santé se continue aujourd'hui sur les ondes de la télévision. Et le message y sera plus cru encore que les imageschocs jusqu'à maintenant publiées dans les quotidiens québécois.

On a vu un médecin bâillonné, une fille intubée. Cette fois les médecins nous plongent en plein cauchemar sanglant, pire que la grisâtre publicité que les infirmières avaient fait diffuser il y a quelques années pour faire prendre conscience de leur importance, et qui avait à l'époque beaucoup choqué.

À la première seconde, le ton est donné : les portes d'une salle d'opération volent sous le choc d'une civière conduite au pas de course. Il y a eu accident. Grave. La jeune blessée de 12 ans à peine saigne d'abondance : déjà la moitié du sang perdu, crie le chirurgien. Il faut de toute urgence stopper l'hémorragie.

Les gros plans sur les blessures, les gestes chirurgicaux, ne sont pas ménagés. Le temps file, la vie aussi. L'anxiété monte, l'infirmière éponge le front du chirurgien.

Changement d'image : un autre homme presse un mouchoir contre sa joue. Un fonctionnaire. Dans un calme bureau. Avec devant lui le projet de loi 120 du ministre Côté qu'il examine et feuillette.

Retour à l'hôpital : "On a au moins du coagulant?", presse le chirurgien. "La demande n'a pas encore été approuvée", répond l'infirmière.

13. J. Boileau, «La propagande des médecins sera plus crue à la télé», Le Devoir [de Montréal] (10 juin 1991) 1; S. Scott, «CBC won't run doctors' TV ad opposing health-care reform», The Gazette [de Montréal] (11 juin 1991) A5. 
Et l'on revoit le fonctionnaire, drapeau du Québec en arrière-plan, prendre sa mallette et quitter la pièce, sa journée faite, avec une copie de la loi 120 dans la main. La bureaucratie, la pire des maladies, conclut la publicité. Soixante secondes ont passé, mais mille allusions ont été envoyées $[\ldots] \gg{ }^{14}$

L'effet de cette publicité a été considérable dans le retournement de situation concernant l'opinion émise par les journalistes à l'égard des médecins. Si la plupart des quotidiens étaient jusque là fort gracieux face au corps médical, un certain scepticisme s'est dès lors emparé des journalistes qui ont commencé à ouvrir leurs colonnes à d'autres acteurs du débat entourant la réforme des services de santé et des services sociaux. Certains de ceux-ci seront d'ailleurs très virulents à l'égard de la campagne publicitaire. Notons le cas des directeurs d'hôpitaux qui, par la voie d'un communiqué de presse, expriment ainsi leur indignation face à cette situation : «C'est excessif, exagéré, abusif, faux et même méprisant.» ${ }^{15}$ Ils allient ainsi leur voix à celle de nombreux groupes qui dénoncent l'omniprésence du discours des médecins dans le débat sur le projet de réforme proposé par le ministre Côté.

Changeant tout à coup d'alliés, les journalistes s'insurgent contre les méthodes utilisées par les médecins pour faire valoir leurs intérêts dans ce dossier. Le Soleil de Québec relate les différents moyens de pression envisagés par le corps médical en décriant chacun de ceux-ci pour leur démagogie trop appuyée : marche des médecins devant le Parlement provincial (29 mai 1991), intervention des médecins spécialistes devant leurs députés respectifs, demande faite aux patients de signer une pétition réclamant le retrait du projet de loi 120, etc... ${ }^{16}$

Qui plus est, les journalistes vont désormais chercher l'opinion des politiciens et des hauts-fonctionnaires qu'ils dénonçaient autrefois pour faire contre-poids à l'offensive publicitaire des médecins. C'est ainsi qu'ils feront grand état des propos de Marc-Yvan Côté concernant la pétition qui circule dans

\footnotetext{
14. J. Boileau, ibid.

15. F. Berger, «La publicité choc des médecins soulève la colère des directeurs d'hôpitaux», $L a$ Presse [de Montréal] (12 juin 1991) A2.

16. L. Lemieux, «Les hôpitaux au ralenti mercredi», Le Soleil [de Québec] (23 mai 1991) A3.
} 
les cabinets de médecin. On peut ainsi lire dans un article de Martha Gagnon, daté du 23 mai 1991, que le ministre trouve :

«(...) pour le moins curieux que ces derniers profitent des consultations à leurs cabinets pour informer les patients de leurs revendications. "Cela démontre le caractère généreux de notre régime. Les médecins seront payés pour informer leurs clients", a-t-il ironisé.» ${ }^{17}$

Dans un billet, Jean Francoeur dénonce la menace de grève des médecins. Il dénonce ce qu'il perçoit comme un traitement de faveur qui bénéficie aux médecins plutôt qu'aux autres travailleurs du secteur sociosanitaire. En ce sens, il s'avoue être contre les avantages que procure la loi 120 aux médecins. Pour Francoeur, cette loi ne fait que renforcer le pouvoir médical et contribue à un meilleur positionnement des médecins dans le système de santé québécois. $^{18}$

Ce qui s'annonçait au départ comme une lutte des divers intervenants en matière de santé au Québec contre une réforme qui ne laisserait pas la part belle aux usagers de ce système socio-sanitaire s'est vite transformé, dans les médias, en un long dialogue entre trois interlocuteurs principaux : les médecins, les représentants de l'État et les journalistes. Trois groupes professionnels qui s'affrontent pour accroître leur zone d'influence auprès de la société civile et s'assurer la possession d'un certain contrôle social.

17. M. Gagnon, «Les médecins mettent une sourdine à leurs pressions», La Presse [de Montréal] (23 mai 1991) A4.

18. J. Francoeur, «La grève des médecins, deux poids deux mesures», Le Devoir [de Montréal] (23 mai 1991) B1. 


\section{L'insertion de points de vue alternatifs dans le discours médiatique : le cas de la Coalition pour la santé et le bien-être}

Bien sûr, d'autres acteurs se sont inscrits dans les délibérations transmises par les médias écrits. Il faut toutefois admettre qu'ils ont été relégués à des rôles de second plan et que leur parole n'a été admise que si elle s'inscrivait dans les enjeux déjà déterminés par les trois groupes mentionnés précédemment. C'est le cas, entre autres, de la Coalition pour la santé et le bien-être [ci-après la Coalition] qui regroupe des organismes populaires, des syndicats, des représentants de consommateurs et de personnes âgées ainsi que des groupes professionnels.

Cette coalition, formée au mois de mai 1991, n'a que très peu retenu l'attention des journalistes jusqu'au jour où les médecins ont engagé leur campagne de publicité. C'est à ce moment bien précis que ce groupe, qui s'oppose aux revendications des médecins, est devenu un acteur ayant pleinement droit au chapitre pour les médias. Lia Lévesque est l'une des premières journalistes à traiter des demandes de la Coalition :

«À leurs yeux, certains articles de la réforme ne doivent pas être négociables, comme la présence de plus de médecins en région, l'accessibilité aux soins, la régionalisation des budgets de la Régie de l'assurance-maladie, le fait que la réforme soit conçue pour l'usager, et non les médecins.» ${ }^{19}$

Bon nombre de journalistes verront dans les revendications de la Coalition une alternative valable à «l'attitude impérialiste» du corps médical. Ainsi acquiescent-ils aux propos des représentants de la Coalition en affirmant que «(...) le ministre de la Santé et des Services sociaux, Marc-Yvan Côté, aurait consenti un compromis de taille en retirant de sa réforme la régionalisation des budgets de la Régie de l'assurance-maladie du Québec.» ${ }^{20}$

19. L. Lévesque, «Une imposante coalition veut dire son mot sur la santé», Le Soleil [de Québec] (21 juin 1991) A7.

20. F. Tremblay, «Entente avec les médecins», Le Soleil [de Québec] (17 août 1991) A1. 
La Coalition, tout comme une bonne part des représentants des médias, dénoncent ce recul qui intervient lors de l'été 1991. Les grands quotidiens laissent une place importante au porte-parole de ce regroupement qui affirme que :

«(...) le maintien de la centralisation du budget de la Régie de l'assurance-maladie à Québec... pourrait compromettre l'un des objectifs majeurs de la réforme, soit celui d'assurer l'accessibilité des soins dans les régions. ${ }^{21}$

Qui plus est, les journalistes s'inquiètent du pouvoir des médecins qui, à l'été 1991, deviennent le seul groupe qui aura eu la chance de négocier de façon privée le projet de loi avec les représentants du ministère de la Santé et des Services sociaux. Des résultats de ces négociations, les membres de la Coalition se disent «(...) très sceptique(s) face au pouvoir et à la place qu'on a donnés aux médecins, à l'intérieur des établissements comme dans tout le système.» ${ }^{22}$ De plus, la Coalition :

«(...) réitère sa demande de création d'un Conseil national de la santé et du bien-être pour se préoccuper de la dimension sociale comme de la dimension santé. Pour faire contrepoids également au tout nouveau Conseil médical du Québec et des Commissions médicales régionales.» ${ }^{23}$

\section{La recherche des adversaires du corps médical}

Le soudain intérêt des médias écrits pour la Coalition n'aura pas raison des demandes des médecins et c'est un peu à une recherche active des adversaires du corps médical que se livrera l'ensemble des journalistes à l'été de 1991. Ils souligneront, par exemple, l'exaspération du président de l'Association des centres d'accueil du Québec, M. Michel Clair, qui regrette la «bataille de

21. F. Tremblay, ibid; voir aussi A. Pépin, «Les médecins font des gains», La Presse [de Montréal] (17 août 1991) A1; G. Boivin, «Très net recul du Ministre de la Santé», Le Soleil [de Québec] (20 août 1991) A8.

22. G. Boivin, ibid.

23. Ibid. 
pouvoir» que les médecins mènent contre les hauts-fonctionnaires - une bataille qui, selon les dires de ce dernier, serait déjà gagnée par les médecins et ne ferait qu'occulter les véritables objectifs de la réforme proposée par Marc-Yvan Côté. ${ }^{24}$

D'autres journalistes mentionneront la difficile relation qu'entretiennent les médecins avec les CLSC, exprimant par ailleurs l'absence d'altruisme et de bonne volonté dans le discours médical. La quasi-absence des médecins dans les CLSC démontre bien, selon Michelle Coudé-Lord, la profonde rupture qui existe entre les politiques de santé émises par le Ministère de la Santé et des Services sociaux [ci-après le MSSS] et les véritables volontés du corps médical :

«50\% des effectifs médicaux sont à peine comblés après bientôt 20 ans d'existence. Pire encore, $50 \%$ des médecins recrutés quittent avant cinq ans! Nous sommes loin de la nouvelle histoire d'amour souhaitée par le ministre Marc-Yvan Côté entre les CLSC et le monde médical! $\gg^{25}$

C'est dans cet esprit que les médias écrits ouvriront leurs pages aux propos et aux revendications de la Fédération des CLSC du Québec, reprenant entre autres, comme c'est le cas du Soleil de Québec, leur communiqué de presse qui appuie la réforme Côté de façon intégrale :

«[U]ne proposition portant sur les services médicaux et la réforme de la santé et des services médicaux a été adoptée à l'unanimité, vendredi, lors de l'assemblée générale annuelle de la Fédération des CLSC. (...) la proposition se lit comme suit : "Que la Fédération des CLSC du Québec réitère son appui au Ministre de la Santé et des Services sociaux, M. Marc-Yvan Côté, dans ses efforts pour réaliser une meilleure répartition des médecins entre les régions et pour les établissements publics, particulièrement en maintenant sa position concernant :

- l'agrément des médecins en cliniques privées;

- la régionalisation des budgets de la Régie de l'assurance-maladie du Québec;

24. Ibid.

25. M. Coudé-Lord, «L'heure de vérité a sonné pour les CLSC», Journal de Québec (2 juin 1991) 14. 
Ces demandes, loin d'être l'apanage de la Fédération des CLSC, sont aussi partagées par de nombreux groupes communautaires, par certains professionnels (ergothérapeutes, psychologues, travailleurs sociaux) et par la grande Coalition. Dans cet esprit, les journalistes ajoutent leur grain de sel en multipliant les reportages qui traitent des bénéfices d'une régionalisation des budgets de la Régie de l'assurance-maladie du Québec [ci-après la RAMQ] et des plans d'effectifs médicaux.

\section{Les négociations de l'été 1991 et le retour de l'usager dans les quotidiens québécois}

Pourtant, toutes ces démarches ne sauront freiner la poussée des médecins, qui prendront pour appui la population grâce à leur imposante campagne de publicité. Les négociations privées entre les médecins et les hautsfonctionnaires du MSSS seront, dès lors, incontournables et le débat sur la réforme du système de santé québécois ne se résumera plus qu'en une lutte entre deux acteurs principaux : l'État, d'une part, et les médecins, de l'autre.

Certains journalistes verront les négociations de l'été 1991 comme une façon de corriger une erreur de stratégie pour les dirigeants du MSSS :

«La négociation qui s'amorce aurait dû se faire dans les semaines ou les mois qui ont précédé la publication du livre blanc et du projet de loi. En faisant l'économie de cette consultation avec la profession médicale, M. Côté se sera attiré bien des désagréments.» ${ }^{27}$

Assez curieusement, c'est à ce moment que certains journalistes ressortiront le citoyen - pourtant censé être au centre de cette réforme - comme argument contre les négociations secrètes qui se trament alors et qui devraient porter fruit à la fin du mois d'août :

26. M. Laferrière, «Réforme de la santé : les CLSC ne céderont pas sur trois points», Le Soleil [de Québec] (2 juin 1991) A1.

27. J. Francoeur, «L'os avec les médecins», Le Devoir [de Montréal] (19 juin 1991) B1 à la p. B2. 
«La nouvelle échéance du 28 août n'offre toutefois aucune garantie sérieuse à la population que le projet remanié protégera ses intérêts. Il est mathématiquement impossible de réviser un projet de loi de 494 articles en deux mois, au coeur de l'été, alors qu'une partie des principaux intervenants seront en vacances. $\rangle^{28}$

Si jusqu'à ce moment la question de la légitimité des médias ne se posait pas (contrairement à celle de l'État ou des médecins), il faut croire que l'annonce de négociations privées entre les médecins et l'État québécois a su réveiller les troupes journalistiques qui ont pris pleinement conscience, dès lors qu'ils se sentaient progressivement écartés du débat, de leur statut latent de groupe de pouvoir.

C'est à ce moment que les médias ont opéré un autre changement de cap qui les a entraînés à cesser de faire l'économie d'une réflexion sur la source de leur légitimité. Dans le duel qui les opposent aux médecins et à l'État, les journalistes ont finalement recentré leurs énergies en fonction du mandat qui leur est conféré, soit celui d'informer l'opinion publique.

N'ayant pas répondu jusque là à la légitimité qui est la leur, ${ }^{29}$ les médias écrits ont à ce moment changé leur fusil d'épaule en assoyant leur discours sur les droits de la population profane qu'ils sont sensé informer. C'est en insérant le points de vue du citoyen ordinaire que les journalistes ont su se positionner dans le débat législatif entourant l'adoption de la loi 120 et faire figure d'autres choses que d'acteur marginal dans ce dossier.

Dès lors s'inscrit donc dans le discours des médias la revendication des droits des usagers qui avait été occultée jusque-là. Lysiane Gagnon, pourtant assez discrète sur la question auparavant, se fera l'une des principales défenderesse de la population :

28. L. Gagnon, «Qui défend le public?», La Presse [de Montréal] (18 juin 1991) B3.

29. Contrairement aux médecins qui n'ont cessé de penser leur discours en tant que responsables de la santé de la population québécoise ou contrairement à l'État qui n'a eu cesse de rappeler son rôle de garant du bien-être général de cette même population, les journalistes n'ont pas pris le relais du discours de la population profane. 
«Dans cette lutte de pouvoir, qu'arrive-t-il au malade, ou, comme on dit dans l'infâme jargon technocratique, au "bénéficiaire"? Pas grand'chose de bon, car par définition, le souci principal de l'administrateur est de gérer son budget d'une manière qui lui vaudra une bonne réputation au ministère. Et tant pis pour les patients quand leurs besoins excèdent les normes.» ${ }^{30}$

Loin d'adopter une position aussi critique que celle de ses autres collègues-journalistes à l'égard des médecins, Lysiane Gagnon persiste dans l'idée que la réforme proposée par le ministre Côté est injustifiée et injustifiable. En ce sens, elle rejoint le rédacteur en chef de L'Actualité, Jean Paré, en épargnant le corps médical pour s'attaquer aux fondements même de cette réforme. Ce dernier avait d'ailleurs déjà donné la couleur de ses opinions beaucoup plus tôt dans l'année en accordant une entrevue à La Tribune de Sherbrooke où il se questionnait sur l'urgence d'un tel remue-ménage dans le domaine de la santé et des services sociaux au Québec :

«Qu'est-ce qui justifie Dieu-le-père-qui-est-à-Québec de jeter le système de santé québécois dans une autre de ses révolutions pas si tranquilles? N'avons-nous pas le meilleur système de santé au monde? Et côté coût, on en est, assure-t-on, à 7\% du PIB? 2\% de moins que le reste du Canada, $4 \%$ de moins que les États-Unis. Jusqu'où veut-on descendre? Jusqu'où veut-on être efficace? Ne suffit-il pas de corriger le cafouillis des urgences, dû à l'incompétence plutôt qu'au manque de moyens? Et se construire des résidences pour malades chroniques et gériatriques?» ${ }^{31}$

Jean Paré, tout comme Lysiane Gagnon et bien d'autres journalistes, reviendront aux objectifs du livre blanc de Marc-Yvan Côté qui plaçait le citoyen au coeur de la réforme pour démontrer l'illogisme de ce face-à-face entre les médecins et le gouvernement du Québec. Les critiques fuseront de toutes parts pour décrier les visées autonomistes des médecins ou les volontés de contrôle du système de santé par les hauts-fonctionnaires. Lorsque la situation l'indique, certains journalistes utiliseront même les propos d'autres acteurs dans

30. L. Gagnon, «Le bon vendeur», La Presse [de Montréal] (25 mai 1991) B3.

31. J. Paré, supra note 8. 
(1997-98) 28 R.D.U.S.

Les médias écrits

et le processus d'émergence

ce débat pour faire valoir leur thèse. C'est le cas ici de Louise Lemieux qui, voulant rétrospectivement défendre le citoyen, s'insurge tout comme la Fédération des infirmières et infirmiers du Québec contre le bras-de-fer qu'ont livré les médecins au gouvernement :

«Non seulement les médecins ont-ils conservé leur pouvoir dans le système de santé, ils l'ont augmenté et consolidé, déplore la Fédération des infirmières et infirmiers du Québec (FIIQ). Ce tour de force, ils l'ont réussi au terme de discussions en catimini, cet été, avec le gouvernement.

Le processus de cet été est inquiétant, et antidémocratique. Le système de santé et de service sociaux est un système public et la population a été tenue à l'écart des négociations entre les médecins et le gouvernement, constate Sylvie Boulanger, vice-présidente de la FIIQ.

Au terme de deux mois de discussions avec les représentants du ministère de la Santé et des Services sociaux, les fédérations de médecins du Québec ont réussi à reprendre ce que leur enlevait le projet de loi $120[\ldots]\rangle^{32}$

Encore une fois, et a posteriori, cet exemple prouve qu'avec la réduction du débat en un affrontement entre le corps médical et les hauts-fonctionnaires, les médias écrits n'ont que très tardivement traité de la place de l'usager dans le système de soins au Québec. Très souvent les journalistes ont pris le relais du discours d'un des deux grands belligérants en espérant, du moins en soumettonsnous l'hypothèse (qui restera à vérifier), tailler leur place dans l'esprit de l'opinion publique.

Comme ces deux acteurs importants, les médias ont concouru à une certaine surenchère langagière autour des droits de l'usager qui n'a pas nécessairement mené à une défense active de ces mêmes droits. Du coup, ils ont aussi joué le jeu de la variable muette «citoyen», mais pour faire plutôt un contre-poids à la polarisation du débat entre les médecins et l'État. L'utilisation

32. L. Lemieux, «Les médecins ont consolidé leur pouvoir dans les hôpitaux», Le Soleil [de Québec] (11 septembre 1991) A3. 
d'un discours axé sur les droits du citoyen n'aura toutefois pas permis d'éviter la phase de négociation privée entre ces deux principaux acteurs de la réforme.

En ce sens, nous pouvons nous questionner sur la véritable place qui a été laissée aux usagers dans les médias. Bien peu de journalistes ont porté attention à ceux-ci avant que les médecins ne préparent leur grande offensive publicitaire. Tout au plus, les commentaires ont été discrets à leur égard, témoignant d'une sympathie qui laisse croire que les différents quotidiens de la province soignent leur lectorat plutôt que d'être préoccupés par une représentation adéquate dans leurs pages de tous les intervenants présents dans ce débat législatif.

Cette timidité des médias face à la situation des usagers est particulièrement perceptible dans les grands éditoriaux rédigés essentiellement par Jean Francoeur (Le Devoir), Agnès Gruda (La Presse), Lysiane Gagnon (La Presse) et les divers rédacteurs de la Gazette. Les charges plus dures à l'endroit de l'État québécois sont peu nombreuses. L'un des rares exemples de critique portées à l'égard de la loi 120 provient de la plume de Jean Francoeur qui s'élève contre la portée réelle de l'article 13 :

«Prenons (sans ironiser) ce fameux droit des usagers qui tient une place démesurée dans le projet de loi 120. Comment ne pas voir que l'article 13, qui soumet l'exercice de ce droit aux dispositions législatives et réglementaires touchant l'organisation des établissements, leur fonctionnement et leurs ressources, constitue en fait une limite beaucoup plus nette que sous la loi actuelle, et pourrait même marquer un recul (souhaitable? discutons-en) tout en facilitant un aménagement plus rationnel des services.» ${ }^{33}$

Il est à noter que ce fameux article 13 restreint sérieusement l'accès aux soins pour les usagers, alors que, dans la même loi, les articles 5 et 6 portent sur la liberté du choix du professionnel ou de l'établissement pour les services que désire recevoir l'usager. Comme bon nombre de journalistes, Francoeur se fera encore plus précis sur la portée de cet article à la fin du processus législatif en 
affirmant que l'organisation structurelle et matérielle du système de santé québécois a primauté sur le droit de ses usagers :

«En ce qui a trait aux droits des usagers (...), le projet de loi améliore le processus d'examen des plaintes. Il réaffirme également le droit de choisir l'établissement ou le professionnel duquel on désire recevoir des services. Mais c'est pour apporter à ce droit une forte sourdine : l'article 13 précise qu'il ne pourra s'exercer qu' "en tenant compte des dispositions législatives et réglementaires relatives à l'organisation et au fonctionnement de l'établissement ainsi que des ressources humaines, matérielles et financières dont il dispose"..» ${ }^{34}$

Ce dégel en faveur des citoyens apparaît bien tardif puisqu'il survient lors de l'été 1991, alors que tout a déjà été pratiquement joué. Pourtant, les divers enjeux qui touchaient de près ou de loin les usagers du système de santé étaient bel et bien présents auparavant : processus d'examen des plaintes, confidentialité du dossier du patient, réduction de l'accessibilité des soins, participation des citoyens aux conseils d'administration des établissements, etc... C'est donc rétrospectivement que les journalistes critiquent le déroulement des discussions entourant l'adoption du projet de loi 120 et les résultats des négociations entre les médecins et les responsables du MSSS. Les propos des journalistes prennent alors toutes les formes du passé et du conditionnel, comme le démontre encore bien l'exemple-type de l'éditorial de Jean Francoeur qui porte sur la participation des citoyens aux divers processus décisionnels dans le système de santé québécois :

«Une véritable réforme aurait mis la hache dans le mythe de la participation et confié tous les sièges du conseil d'administration à des personnes de l'extérieur, aux citoyens et aux citoyens seulement. (...) Les citoyens devront se contenter d'être majoritaires au sein des conseils dont ne seront pas exclus les salariés de l'établissement ni, bien sûr, le médecin, entrepreneur libre.» ${ }^{35}$

34. J. Francoeur, «Le trac», Le Devoir [de Montréal] (26 août 1991) 12.

35. J. Francoeur, ibid. 
Que ce soit pour la question de la participation ou pour celle de la confidentialité du dossier du patient, ${ }^{36}$ les représentants des médias écrits s'avèrent être beaucoup plus sceptiques quant aux objectifs du projet de loi, une fois celui-ci adopté. Dans ce retour en force du citoyen au fur et à mesure que la future loi 120 prend forme, on peut se demander si les médias écrits n'ont finalement réalisé que très tardivement l'importance de la variable «usager» dans leur discours ou s'ils n'ouvrent tout simplement pas la brèche pour des débats qui resteront à venir, cette nouvelle loi suscitant encore beaucoup d'interrogations après son adoption.

Le constat sévère d'une loi imparfaite et l'ouverture soudaine sur le droit des usagers pourraient laisser croire que les médias ont aussi été emportés par ce qu'ils ont critiqué chez les principaux acteurs de cette réforme de la santé et des services sociaux, à savoir une «démagogie outrancière» qui ne fait que servir les intérêts de ceux qui la privilégient.

En ouvrant à nouveau le débat sur les droits des citoyens, une fois la loi 120 adoptée, les médias écrits préparent le terrain pour d'autres affrontements en s'appuyant cette fois-ci sur ce qui constitue essentiellement son lectorat, la population profane du Québec. En interpellant aussi directement leur public, les médias écrits maintiennent ainsi la tension qui, depuis, ne s'est que très peu relâchée sur le système de santé québécois.

\section{CONCLUSION}

L'étude du rôle des médias écrits dans le processus d'élaboration, de discussion et d'adoption de la loi 120 nous aura permis de cerner comment ceuxci procèdent dans la sélection du contenu informatif qui tapisse leurs pages. Qui plus est, l'analyse du contenu journalistique nous aura aussi permis de saisir quels sont les groupes sociaux qui ont plus particulièrement droit au chapitre dans les quotidiens québécois.

36. Agnès Gruda se fait d'ailleurs l'une des principales critiques du projet à cet égard, notamment dans un virulent éditorial publié à la mi-juin 1991 : Agnès Gruda, «La confidentialité des dossiers médicaux menacée», La Presse [de Montréal] (13 juin 1991) B2. 
De façon générale, nous pouvons affirmer que les médias écrits accordent de l'importance aux groupes qui :

- sont bien circonscrits (dont on peut tracer les frontières aisément);

- disposent de ressources (humaines et matérielles) importantes;

- possèdent une influence et un pouvoir certain sur l'opinion publique;

- adoptent un discours cohérent et univoque.

Dans cet esprit, il n'est pas étonnant qu'un groupe aussi large que celui des usagers n'ait pas la place qui devrait normalement lui être accordée si l'on se fiait sur des critères purement démographiques. L'usager, ou le citoyen, fait partie d'une catégorie sociale beaucoup trop diffuse pour qu'on puisse la cerner aisément. De plus, cette catégorie n'est pas exclusive. Nous pourrions prendre comme exemple le cas du médecin qui est, tout à la fois, producteur de services mais aussi usager du système, pour illustrer ce que nous avançons. Mais nous pouvons aussi ajouter que la catégorie «usager» est tellement hétérogène qu'on ne peut arriver à dresser un portrait de celle-ci sur la base de quelques variables réduites. À vrai dire, les variables qui permettraient de définir ce groupe social sont infinies : âge, sexe, occupation professionnelle, origine ethnique, lieu d'habitation, habitudes de consommation, degré de sédentarisation, etc... La liste serait longue et, en bout du compte, elle ne ferait que mieux nous informer sur ce que nous démontrons déjà, à savoir que cette multitude de variables finit par refléter la pluralité des intérêts qui sont latents dans ce vaste groupe.

Cette pluralité des intérêts entraîne une difficulté majeure pour ce groupe, celle de la mobilisation des ressources aux fins de la poursuite d'un objectif commun. Face à des intérêts si différents au sein d'un même groupe, les logiques d'action individuelles prennent le pas sur la définition d'un devenir collectif. Dans ce contexte, l'élaboration d'un discours cohérent qui saurait réunir toutes les parties de ce groupe tient d'un héroïsme et d'une témérité toute don quichottesque.

Le groupe social «citoyen» n'est en fait qu'un alibi pour laisser croire au caractère démocratique du processus juridique mis à l'oeuvre dans la réforme de la santé et des services sociaux. Nous sommes tous des citoyens; que nous soyons fonctionnaires, plombiers ou médecins, nous inscrivons tous notre expérience personnelle dans le cadre juridique, politique et économique d'un 
État moderne. En ce sens, nous pouvons tous nous définir, comme le traduit joliment l'expression populaire, comme des «Monsieur-et-madame-tout-lemonde». De cette appellation, nous pouvons dire qu'elle ne nous permet pas de se hisser au-delà de la société civile et d'acquérir un certain pouvoir ou du prestige social. Il faut aller chercher dans les autres rôles qui nous sont attribués les ferments d'une certaine influence sociale.

C'est des discours et des intérêts liés à ces autres rôles que les médias écrits font leurs choux gras. Toutefois, les journalistes, comme bien d'autres groupes sociaux, ne sont pas exempts des velléités de prestige social. Ils participent à cette quête de pouvoir et se laissent prendre au jeu des luttes d'intérêts. Pour être reconnu comme un joueur aguerri et rusé, le journaliste doit savoir utiliser des variables aussi abstraites et mystérieuses que celle du citoyen. Des variables qui interpellent le plus grand nombre sans qu'on ne puisse vraiment clairement en définir les contours.

Dans le débat qui a entouré le projet de loi 120, les droits du citoyen ont toujours occupé une place dans les divers discours rapportés par les médias écrits québécois. Ce n'est pourtant qu'à la toute fin de ce débat que les journalistes ont accentué leur analyse de ces droits, prenant partie pour ce citoyen si large et si difficilement identifiable, pour enfin annoncer que la discussion ne serait pas close avec l'adoption de ce projet de loi. Ce faisant, ils ont interpellé une majorité de lecteurs qu'ils ont pris pour alliés dans leur quête de pouvoir et de prestige. En tentant de tailler leur place sur cette scène hautement politique, ils ont ravivé de plus belle les luttes d'intérêts qui persistent encore aujourd'hui dans notre système de santé et de services sociaux et dont, il nous semble, nous n'avons pas fini de voir les répercussions. 